\title{
CONWAY ALGEBRAS AND SKEIN EQUIVALENCE OF LINKS
}

\author{
JÓZEF H. PRZYTYCKI AND PAWEL TRACZYK
}

\begin{abstract}
We consider a class of pairs of links which are not skein equivalent but have the same invariant in every Conway algebra.
\end{abstract}

1. Conway algebras. We will first recall the notion of Conway algebra as introduced in $[\mathbf{P T}]$.

DEFINITION 1.1. A Conway algebra is an algebra $A$ with a sequence of 0 argument operations $a_{1}, a_{2}, \ldots$ and two 2 -argument operations $\mid$ and $*$, which satisfy the following conditions:

C1. $a_{n} \mid a_{n+1}=a_{n}$,

C2. $a_{n} * a_{n+1}=a_{n}$ (C1 and $\mathrm{C} 2$ are initial conditions properties),

C3. $(a \mid b)|(c \mid d)=(a \mid c)|(b \mid d)$,

C4. $(a \mid b) *(c \mid d)=(a * c) \mid(b * d)$ (C3, C4 and C5 are transposition properties),

C5. $(a * b) *(c * d)=(a * c) *(b * d)$,

C6. $(a \mid b) * b=a$,

C7. $(a * b) \mid b=a$.

As shown in [PT] every Conway algebra yields an invariant of links which is constant on skein equivalence classes (skein invariant). It is uniquely determined by the following conditions:

$A_{T_{n}}=a_{n}$ (initial relations),

$A_{L_{+}}=A_{L_{-}} \mid A_{L_{0}}$ and $A_{L_{-}}=A_{L_{+}} * A_{L_{0}}$ (Conway relations).

Here $T_{n}$ denotes a trivial link of $n$ components and $L_{+}, L_{-}$, and $L_{0}$ are diagrams of oriented links identical except near one crossing point (see Figure 1.1).

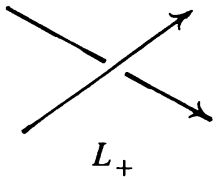

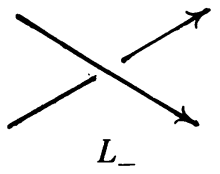

FIGURE 1.1

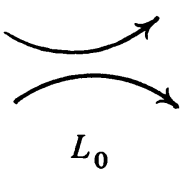

$L_{\mathbf{0}}$

2. Skein equivalence and the signature. We recall that skein equivalence is the smallest equivalence relation $\sim_{s}$ on the set of all oriented links in $S^{3}$ such that:

(i) if $L$ is ambient isotopic to $L^{\prime}$, then $L \sim_{s} L^{\prime}$,

Received by the editors December 10, 1985 and, in revised form, April 8, 1986.

1980 Mathematics Subject Classification (1985 Revision). Primary 57M25.

Key words and phrases. Skein equivalence, Jones polynomial, signature.

The second author acknowledges support received during the preparation of this paper from the Matsumae International Foundation and Osaka University. 
(ii) if $\left(L_{+}, L_{-}, L_{0}\right)$ and $\left(L_{+}^{\prime}, L_{-}^{\prime}, L_{0}^{\prime}\right)$ are skein triples, then

(a) $L_{+} \sim_{s} L_{+}^{\prime}$ and $L_{0} \sim_{s} L_{0}^{\prime}$ implies $L_{-} \sim_{s} L_{-}^{\prime}$, and

(b) $L_{-} \sim_{s} L_{-}^{\prime}$ and $L_{0} \sim_{s} L_{0}^{\prime}$ implies $L_{+} \sim_{s} L_{+}^{\prime}$.

In this section we will give examples of links which cannot be distinguished by any invariant yielded by a Conway algebra but which are not skein equivalent. The examples to be considered were originally constructed by Birman [B], and Lozano and Morton in order to provide different knots with the same 2-variable Jones polynomial. It was observed by Lickorish and Millet [LM] that these knots are not skein equivalent, because they have different signatures.

It was (more or less) shown by Conway [C] (also Giller [G]) that the signature of knots is a skein equivalence invariant. It can be shown in a more general context: the Tristram-Levine signature is related to the Conway polynomial in just the same way as classical signature to the determinant invariant. One can hope for an analogous invariant related to 2 -variable Jones polynomial.

3. Equivalences in the Conway algebra. We will now show that there are nonskein equivalent knots which cannot be distinguished by any invariants yielded by any Conway algebra. We will use notation and terminology of Murasugi [M].

THEOREM 3.1. Let $\gamma$ be a 3-string braid $\sigma_{1}^{a_{1}} \sigma_{2}^{a_{2}} \cdots \sigma_{1}^{a_{2 k-1}} \sigma_{2}^{a_{2 k}}$ such that the sum of the exponents of $\gamma, e(\gamma)=\sum_{i=1}^{2 k} a_{i}$, is equal to 0 . Then the closed braid $\hat{\gamma}$ cannot be distinguished from its mirror image $\hat{\bar{\gamma}}$ by the invariant yielded by any Conway algebra.

PROOF. Consider a Conway algebra $\left(A, a_{1}, a_{2}, \ldots, \mid, *\right)$. First we formulate a lemma which is crucial to our proof of the theorem, then show how the theorem follows from the lemma, and prove the lemma .

We use the following notation: if $\gamma=\gamma_{1} \sigma_{i}^{a} \gamma_{2} \sigma_{j}^{b} \gamma_{3}$ is a 3-braid, then $A_{a+p, b+q}$ denotes the value of the invariant of the closed braid $\hat{\gamma}_{a+p, b+q}$, where $\gamma_{a+p, b+q}=$ $\gamma_{1} \sigma_{i}^{a+p} \gamma_{2} \sigma_{j}^{b+q} \gamma_{3}$. Strictly speaking $\gamma_{1}, \gamma_{2}, \gamma_{3}, i$, and $j$ should be given explicitly in the notation, but we adopt a rather informal convention of treating $a$ and $b$ as recognizing signs for them. We will also use a natural convention of writing $\gamma_{-b,-a}$ for the mirror image of $\gamma_{a, b}$.

We have the following obvious equalities:

$$
\begin{aligned}
& A_{a, b}=A_{a-2, b}\left|A_{a-1, b}=\left(A_{a-2, b+2} * A_{a-2, b+1}\right)\right| A_{a-1, b}, \\
& A_{c, d}=A_{c, d+2} * A_{c, d+1}=\left(A_{c-2, d+2} \mid A_{c-1, d+2}\right) * A_{c, d+1} .
\end{aligned}
$$

Let us formulate our lemma.

LEMMA 3.2. If $A_{a-2, b+2}=A_{c-2, d+2}$ and $A_{a-3, b+3}=A_{c-3, d+3}$, then we have the following equivalence:

$$
\left(w_{2} * A_{a-2, b+1}\right) \mid h_{2}=\left(w_{1} \mid A_{c-1, d+2}\right) * h_{1}
$$

if and only if

$$
\left(w_{2} * A_{a-4, b+3}\right) \mid h_{2}=\left(w_{1} \mid A_{c-3, d+4}\right) * h_{1},
$$

where $w_{1}, w_{2}, h_{1}, h_{2} \in A$.

First we show how to prove Theorem 3.1 using Lemma 3.2. Let $\gamma$ be a cyclically reduced word $\gamma=\sigma_{1}^{a_{1}} \sigma_{2}^{a_{2}} \cdots \sigma_{1}^{a_{2 k-1}} \sigma_{2}^{a_{2 k}}$ with $\left|a_{i}\right|>0$ and $e(\gamma)=0$ (the sum of exponents). We define the complexity of $\gamma$ to be $c(\gamma)=\operatorname{cr}(\gamma)-2 k+p(\gamma)$, where 
$\operatorname{cr}(\gamma)$ is the sum of absolute values of exponents of $\gamma$ (i.e. the number of crossing points of the closed braid $\hat{\gamma}$ ) and $p(\gamma)$ is the number of pairs of exponents $a_{i}, a_{i+1}$ in $\gamma$ having the same sign (in the cyclic word, we consider also the pair $a_{2 k}, a_{1}$ ). We will prove the theorem by induction first on $\operatorname{cr}(\gamma)$, then on $c(\gamma)$. The theorem is obviously true for $\operatorname{cr}(\gamma)=0$. For $c(\gamma)=0$, we have $\hat{\gamma}$ isotopic to its mirror image, since $\gamma$ is a cyclic word of the form $1,-1,1,-1, \ldots$ in this case.

It is easy to see that if $\operatorname{cr}(\gamma)$ and $c(\gamma)>0$ for a cyclic word $\gamma$, then we can choose $a$ and $b$, two of the exponents of $\gamma$, in such a way that either

(a) $\operatorname{cr}\left(\gamma_{a-2, b+2}\right)<\operatorname{cr}(\gamma)$, or

(b) $\operatorname{cr}\left(\gamma_{a-2, b+2}\right)=\operatorname{cr}(\gamma)$ and $c\left(\gamma_{a-2, b+2}\right)<c(\gamma)$.

In both cases we have, by the inductive assumption, $A_{a-2, b+2}=A_{-b-2,-a+2}$ (according to the adopted notation, $A_{-b-2,-a+2}$ is the value of the invariant for the mirror image of $\left.\hat{\gamma}_{a-2, b+2}\right)$. We also have

$$
\begin{aligned}
A_{a, b} & =\left(A_{a-2, b+2} * A_{a-2, b+1}\right) \mid A_{a-1, b}, \\
A_{-b-a} & =\left(A_{-b-2,-a+2} \mid A_{-b-1,-a+2}\right) * A_{-b,-a+1},
\end{aligned}
$$

and we want to prove $A_{a, b}=A_{-b,-a}$.

Let us consider the cyclic word $\gamma_{a-2, b+1}$. We have either

(a) it consists of one letter, and then $A_{a-2, b+1}=A_{-b-1,-a+2}=a_{2} \in A$, and we need to prove the equality

$$
\left(w * a_{2}\right) \mid A_{a-1, b}=\left(w \mid a_{2}\right) * A_{-b,-a+1}
$$

(where $w=A_{a-2, b+2}=A_{-b-2,-a+2}$ ), or

(b) it has exponents $p$ and $q$ such that $|p| \geq 2$ and $p \cdot q<0$.

We will consider the case $p \geq 2$ and $q \leq-1$. We will now use symbols such as $A_{p+x, q+y}$ for values of the invariant for closed braids obtained from $\gamma_{a-2, b+1}$ (not from $\gamma$ !) by changing the exponents $p$ and $q$. We also use $\gamma_{p+x, q+x}$ for the related braids. Using this notation we obtain $A_{a-2, b+1}=A_{p, q}$, and by the inductive assumption

$$
A_{p, q+1}=A_{-q-1,-p}, \quad A_{p-1, q+2}=A_{-q-2,-p+1},
$$

because $\operatorname{cr}\left(\gamma_{p, q+1}\right), \operatorname{cr}\left(\gamma_{p-1, q+2}\right)<\operatorname{cr}(\gamma)$ and $e\left(\gamma_{p, q+1}\right)=e\left(\gamma_{p-1, q+2}\right)=0$.

We are now in position to apply Lemma 3.2 taking $a=p+2, b=q-1, c=-q+1$, $d=-p-2, w_{1}=w_{2}=A_{a-2, b+2}=A_{-b-2,-a+2}, h_{2}=A_{a-1, b}$, and $h_{1}=A_{-b,-a+1}$. We obtain the following equivalence:

$$
\left(w_{2} * \mathcal{A}_{p, q}\right) \mid h_{2}=\left(w_{1} \mid \mathcal{A}_{-q,-p}\right) * h_{1}
$$

if and only if

$$
\left(w_{2} * A_{p-2, q+2}\right) \mid h_{2}=\left(w_{1} \mid A_{-q-2,-p+2}\right) * h_{1} .
$$

We can repeat the procedure until we are reduced to proving (3.3). The same argument works for $p \geq 1$ and $q \leq-2$. The only change is that we are able in this case to diminish $|q|$, not $|p|$. In order to prove (3.3) let us consider equalities

$$
\left(w * A_{a-1, b}\right) \mid A_{a-1, b}=w=\left(w \mid A_{-b,-a+1}\right) * A_{-b,-a+1},
$$

which are true by $\mathrm{C} 6$ and C7. Applying Lemma 3.2 in a manner similar to the above we obtain a sequence of equivalent equalities ending with (3.3), and thus (3.3) is true, which completes the proof of Theorem 3.1. 
It remains to prove Lemma 3.2. Consider an equality

$$
\left(w_{2} * A_{a-2, b+1}\right) \mid h_{2}=\left(w_{1} \mid A_{c-1, d+2}\right) * h_{1} .
$$

Multiply both sides of (3.4) by $\left.\mid h_{1}\right) * A_{c-1, d+2}$. We get an equality equivalent to (3.4):

$$
\left(\left(\left(w_{2} * A_{a-2, b+1}\right) \mid h_{2}\right) \mid h_{1}\right) * A_{c-1, d+2}=w_{1}
$$

(we applied $\mathrm{C} 7$ and $\mathrm{C} 6$ to the right side).

We will now consider a series of equalities obtained by transforming the formula for the left side of (3.5). This will be done by applying the transposition properties, C6 and C7. For the reader's convenience the axiom applied will always be marked. In case of transpositions we will also mark the elements to be transposed by underlining them. The left side of (3.5) is equal to (we begin by replacing $A_{c-1, d+2}$ with $\left.A_{c-3, d+2} \mid A_{c-2, d+2}\right)$

$$
\begin{aligned}
& {\left[\left(\left(w_{2} *\left(A_{a-2, b+3} * A_{a-2, b+2}\right)\right) \mid h_{2}\right) \mid \underline{h_{1}}\right] *\left[\underline{A_{c-3, d+2}} \mid A_{c-2, d+2}\right]} \\
& \stackrel{\mathrm{C4}}{=}\left(\left(\left(w_{2} *\left(A_{a-2, b+3} * A_{a-2, b+2}\right)\right) \mid h_{2}\right) * A_{c-3, d+2}\right) \mid\left(h_{1} * A_{c-2, d+2}\right) \\
& \stackrel{\mathrm{C} 7}{=}\left(\left[\left(w_{2} *\left(A_{a-2, b+3} * A_{a-2, b+2}\right)\right) \mid \underline{h_{2}}\right] *\left[\left(\underline{\left(A_{c-3, d+2} * A_{c-2, d+2}\right.}\right) \mid A_{c-2, d+2}\right]\right) \\
& \mid\left(h_{1} * A_{c-2, d+2}\right) \\
& \left.\stackrel{\mathrm{C4}}{=}\left(\left[\left(w_{2} * \underline{\left(\underline{A_{a-2, b+3}} * A_{a-2, b+2}\right.}\right)\right] *\left[\underline{A_{c-3, d+2} * A_{c-2, d+2}}\right]\right) \mid\left(h_{2} * A_{c-2, d+2}\right)\right) \\
& \text { I }\left(h_{1} * A_{c-2, d+2}\right) \\
& \stackrel{\mathrm{C} 5}{=}\left(\left[\left(w_{2} * A_{c-3, d+2}\right) *\left(\left(\underline{\left.A_{a-2, b+3} * A_{a-2, b+2}\right) * A_{c-2, d+2}}\right)\right] \underline{h_{2}} * A_{c-2, d+2}\right]\right) \\
& \text { I }\left(h_{1} * A_{c-2, d+2}\right) \\
& \stackrel{\mathrm{C} 4}{=}\left(\left(\left(w_{2} * A_{c-3, d+2}\right) \mid h_{2}\right) *\left(\left(\left(A_{a-2, b+3} * A_{a-2, b+2}\right) * A_{c-2, d+2}\right) \mid A_{c-2, d+2}\right)\right) \\
& \mid\left(h_{1} * A_{c-2, d+2}\right) \\
& \stackrel{\mathrm{C} 7}{=}\left[\left(\left(w_{2} * A_{c-3, d+2}\right) \mid h_{2}\right) *\left(\underline{A_{a-2, b+3}} * A_{a-2, b+2}\right)\right] \mid\left[\underline{h_{1}} * A_{c-2, d+2}\right] \\
& \stackrel{\mathrm{C4}}{=}\left(\left(\left(w_{2} * A_{c-3, d+2}\right) \mid h_{2}\right) \mid h_{1}\right) *\left(\left(A_{a-2, b+3} * A_{a-2, b+2}\right) \mid A_{c-2, d+2}\right) \text {. }
\end{aligned}
$$

But we have assumed $A_{a-2, b+2}=A_{c-2, d+2}$, so applying $\mathrm{C} 7$ we obtain

$$
\left(\left(\left(w_{2} * A_{c-3, d+2}\right) \mid h_{2}\right) \mid h_{1}\right) * A_{a-2, b+3}
$$

equal to the left side of (3.5). Thus (3.4) is equivalent to

$$
\left(w_{2} * A_{c-3, d+2}\right) \mid h_{2}=\left(w_{1} \mid A_{a-2, b+3}\right) * h_{1} .
$$

If we repeat the above argument once more we will get Lemma 3.2.

It should be mentioned that we can work similarly with links from Proposition 2 and Lemma 4 of $[\mathbf{B}]$.

If $\gamma$ in Theorem 3.1 has the normal form $\Delta^{2 n} \gamma_{0}(n \neq 0)$ and $\gamma \in \Omega_{6}[\mathbf{M}]$, then $\hat{\gamma}$ has nonzero signature $[\mathbf{M}, \mathbf{B}]$, since it can be shown that $\varepsilon$ from $[\mathbf{M}$, Proposition 11.1] is equal to 0 .

Furthermore, the determinants of links from $\Omega_{6}$ are not zerc, so the signature of these links is a skein invariant. Therefore Theorem 3.1 gives a class of pairs of links which are not skein equivalent but have the same invariant in every Conway algebra. 


\section{REFERENCES}

[B] J. Birman, On the Jones polynomial of closed 3-braids, Invent. Math. 81 (1985), 287-294.

[C] J. H. Conway, An enumeration of knots and links, and some of their algebraic properties, Computational Problems in Abstract Algebra, Pergamon Press, Oxford and New York, 1969, pp. 329-358.

[FYHLMO] P. Freyd, D. Yetter, J. Hoste, W. B. R. Lickorish, K. Millet, and A. Ocneanu, A new polynomial invariant of knots and links, Bull. Amer. Math. Soc. 112 (1985), 239-249.

[G] C. Giller, A family of links and the Conway calculus, Trans. Amer. Math. Soc. 270 (1982), 75-109.

[Go] C. McA. Gordon, Some aspects of classical knot theory, Knot Theory, Lecture Notes in Math., Vol. 685, Springer-Verlag, Berlin and New York, 1978, pp. 1-160.

[J] V. F. R. Jones, A polynomial invariant for knots via von Neuman algebras, Bull. Amer. Math. Soc. 12 (1985), 103-111.

[K] L. Kauffman, The Conway polynomial, Topology 20 (1980), 101-108.

[LM] W. B. R. Lickorish and K. C. Millet, A polynomial invariant of oriented links, Topology 26 (1987), 107-141.

[M] K. Murasugi, On closed 3-braids, Mem. Amer. Math. Soc. No. 151 (1974).

[PT] J. H. Przytycki and P. Traczyk, Invariants of links of Conway type, Kobe J. Math. (to appear).

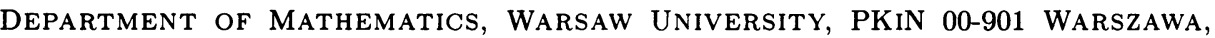
POLAND (Current address of both authors)

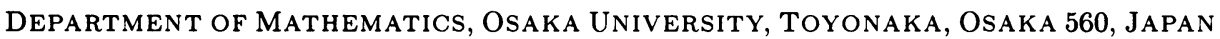

